\title{
Recombination-stable multimeric green fluorescent protein for characterization of weak promoter outputs in Saccharomyces cerevisiae
}

Rugbjerg, Peter; Knuf, Christoph; Förster, Jochen; Sommer, Morten Otto Alexander

Published in:

FEMS Yeast Research

Link to article, DOI:

$10.1093 /$ femsyr/fov085

Publication date:

2015

Document Version

Publisher's PDF, also known as Version of record

Link back to DTU Orbit

Citation (APA):

Rugbjerg, P., Knuf, C., Förster, J., \& Sommer, M. O. A. (2015). Recombination-stable multimeric green fluorescent protein for characterization of weak promoter outputs in Saccharomyces cerevisiae. FEMS Yeast Research, 15(8), [fov085]. https://doi.org/10.1093/femsyr/fov085

\section{General rights}

Copyright and moral rights for the publications made accessible in the public portal are retained by the authors and/or other copyright owners and it is a condition of accessing publications that users recognise and abide by the legal requirements associated with these rights.

- Users may download and print one copy of any publication from the public portal for the purpose of private study or research.

- You may not further distribute the material or use it for any profit-making activity or commercial gain

- You may freely distribute the URL identifying the publication in the public portal 

protein for characterization of weak promoter outputs in Saccharomyces cerevisiae

\title{
Peter Rugbjerg, Christoph Knuf, Jochen Förster and Morten O. A. Sommer*
}

\author{
Novo Nordisk Foundation Center for Biosustainability, Technical University of Denmark, Kogle Allé 6, \\ DK-2970 Hørsholm, Denmark \\ *Corresponding author: Novo Nordisk Foundation Center for Biosustainability, Technical University of Denmark, Kogle Allé 6, DK-2970 Hørsholm, \\ Denmark. Tel: +45 2151 8340; E-mail: msom@bio.dtu.dk \\ One sentence summary: By fusing three different green fluorescent proteins, the molecular brightness becomes high enough to detect low copy \\ numbers inside a yeast cell in a stable manner. \\ Editor: Pascale Daran-Lapujade
}

\begin{abstract}
Green fluorescent proteins (GFPs) are widely used for visualization of proteins to track localization and expression dynamics. However, phenotypically important processes can operate at too low expression levels for routine detection, i.e. be overshadowed by autofluorescence noise. While GFP functions well in translational fusions, the use of tandem GFPs to amplify fluorescence signals is currently avoided in Saccharomyces cerevisiae and many other microorganisms due to the risk of loop-out by direct-repeat recombination. We increased GFP fluorescence by translationally fusing three different GFP variants, yeast-enhanced GFP, GFP+ and superfolder GFP to yield a sequence-diverged triple GFP molecule 3vGFP with 74-84\% internal repeat identity. Unlike a single GFP, the brightness of 3vGFP allowed characterization of a weak promoter in S. cerevisiae. Utilizing 3vGFP, we further engineered a less leaky $\mathrm{Cu}^{2+}$-inducible promoter based on CUP1. The basal expression level of the new promoter was approximately $61 \%$ below the wild-type CUP1 promoter, thus expanding the absolute range of $\mathrm{Cu}^{2+}$-based gene control. The stability of 3vGFP towards direct-repeat recombination was assayed in S. cerevisiae cultured for 25 generations under strong and slightly toxic expression after which only limited reduction in fluorescence was detectable. Such non-recombinogenic GFPs can help quantify intracellular responses operating a low copy number in recombination-prone organisms.
\end{abstract}

Keywords: signal amplification; synthetic biology; promoter engineering; protein multimerization

\section{INTRODUCTION}

Green fluorescent protein (GFP) is an invaluable tool for real-time visualization of intracellular proteins. Since the initial cloning, numerous improvements, variants and applications have been developed (Snapp 2009; Miyawaki 2011). GFP is particularly useful for quantification of intracellular events, localizations and populations at single-cell resolution. However, a minimal expression level is required such that the fluorescent output ex- ceeds the cell autofluorescence and produces detectable signals. Still, biologically important processes occur through the interaction of a few molecules per cell, which is hard to quantify using existing fluorescent proteins and non-specialized experimental setups (Raj and van Oudenaarden 2009; Li and Xie 2011; Gahlmann and Moerner 2014). Further, the engineering of synthetic cell functionalities can depend on fine characterization and balancing of low gene expression levels (Ajikumar et al. 2010; Harton, Wingler and Cornish 2013). 
The strategies for improving fluorescent output signals include the design of new GFP variants such as GFP+, yeastenhanced GFP (yEGFP) and superfolder GFP (sfGFP) (Cormack, Bertram and Egerton 1997; Scholz et al. 2000; Pédelacq et al. 2006). Still, monitoring of single-molecule events such as chromosome movements in Escherichia coli has e.g. required multimerization of 96 DNA-binding sites to localize enough fluorescent protein to produce a distinguishable signal (Xie et al. 2008). Artificial tethering of a bright yellow fluorescent protein (Venus YFP) to the inside E. coli cell membrane allowed a microscope-detectable signal from a single YFP-tagged protein (Yu et al. 2006). Thus, without techniques for single-molecule GFP sensitivity, the fullgenome mapping of subcellular protein localization in Saccharomyces cerevisiae (yeastGFP) did not produce signals above background for 361 open reading frames (8 pct. of total) otherwise shown to be expressed in the growth phase assayed (Ghaemmaghami et al. 2003; Huh et al. 2003). Equivalently, the issue of not detecting all low-expressing $S$. cerevisiae proteins was also observed when the GFP library was applied to flow cytometry (Newman et al. 2006).

In some contexts, simple overexpression may shed light over the lacking information, but since the location of many proteins is a result of interactions with other cell components, a radical change in copy number could easily result in artificial observations. In other situations, the target output is the activity of specific weak promoters, e.g. in synthetic biological circuits, fluorescence-coupled biosensors or when developing promoter libraries. Several technologies permit the engineering of new promoters, e.g. responsive to other inducer molecules by hybridizing with upstream TF-binding sites (Blazeck and Alper 2013) or tuned to match fine, desirable transcription levels through mutagenesis of a strong native promoter (Nevoigt et al. 2006). Difficulties in GFP detection may have been a limitation in these developments for weaker promoter levels, though low expression may be phenotypically important for a wide range of synthetic biology purposes. In synthetic circuit designs, any concealed information on the shape of dose-response curves inhibits the analysis of mechanistic clues otherwise given by the response curvature (Ang, Harris and Hussey 2013). In applications of metabolite biosensors, background-covered signal levels means that the full regulatory capability cannot be utilized, e.g. limiting subsequent fluorescence-activated cell sorting (FACS). Ultimately, such autofluorescence could conceal properly functional GFP completely (Billinton and Knight 2001).

The efforts aimed at reducing the autofluorescence target two phenomena: simple medium autofluorescence arises from measuring fluorescence without isolating cells from medium, e.g. in continuously growing cultures. These effects can be reduced by the choice of medium or spectral unmixing by correcting for autofluorescence from a wavelength representing effects of the culture medium (Lichten et al. 2014). However, the cell autofluorescence is a more central issue, i.a. resulting from the fluorescence of flavins and NAD(P)H (Billinton and Knight 2001). Cellular autofluorescence also impacts techniques such as flow cytometry and microscopy and the weak signal intensity must be amplified intrinsically to the cell.

Previous studies in mammalian cell lines have tackled the obstacle of cell autofluorescence using directly repeated GFPs typically fused three to six times in tandem using a small translational linker (Genové, Glick and Barth 2005). By such approaches, it has been possible to achieve good linear increments in fluorescence signals. However, tandem repeats are problematic in organisms with proficient homologous recombination such as E. coli or S. cerevisiae where recombination between DNA can happen within windows of identity at around 25 nucleotides (Ahn et al. 1988). This could explain why tandem GFP methods are avoided in these organisms. However, even slight sequence divergence between repeats substantially decreases the rate of recombination as seen in the case of recombination between 350 bp inverted repeats, which was 4600 -fold reduced when sequence identity was reduced from $100 \%$ to $74 \%$ in S. cerevisiae (Datta et al. 1997). Similar effects occur in E. coli where up to 1000 -fold reduction was observed following a reduction in repeat identity to $80 \%$ (Rayssiguier, Thaler and Radman 1989).

Thus, in this study we present a simple methodology to take advantage of the ability to add sequence divergence to tandem proteins while maintaining function through variation in amino acid sequence as well as synonymous codon usage. By fusing three different GFP variants that vary mainly at nucleotide level, we produce a new triple tandem GFP (3vGFP) stabilized towards direct-repeat recombination. We demonstrate the utility of 3vGFP through a genetically triggered promoter (ON/OFF) and developing and characterizing a new version of a $\mathrm{Cu}^{2+}$-responsive promoter with reduced leakiness. Application of 3vGFP allowed visualization of weak signals that could not be separated from autofluorescence levels using the brightest individual GFP variant, superfolder GFP. Lastly, we test the stability towards recombination after culturing of the strain harboring 3vGFP through 25 generations.

\section{MATERIALS AND METHODS}

\section{Materials}

Unless otherwise stated, reagents were purchased from SigmaAldrich. Synthetic complete (SC) medium was prepared from $1.4 \mathrm{~g} \mathrm{~L}^{-1} \mathrm{SC}$ drop-out mix lacking uracil, tryptophan, leucine and histidine (Y2001), $6.7 \mathrm{~g} \mathrm{~L}^{-1}$ yeast nitrogen base without amino acids (Y0626) and $20 \mathrm{~g} \mathrm{~L}^{-1} \mathrm{D}$-glucose, $\mathrm{pH}$ standardized to 5.6. When SC was supplemented with additional amino acids, $60 \mathrm{mg} \mathrm{L}^{-1}$ leucine, $20 \mathrm{mg} \mathrm{L}^{-1}$ uracil, $20 \mathrm{mg} \mathrm{L}^{-1}$ histidine-HCl and $20 \mathrm{mg} \mathrm{L}^{-1}$ tryptophan was added. Yeast Peptone Dextrose medium contained $20 \mathrm{~g} \mathrm{~L}^{-1} \mathrm{D}$-glucose.

Oligonucleotides were purchased from Integrated DNA Technologies.

\section{Plasmids}

The plasmids employed in this study are listed in Table 1.

\section{Strains}

The strains analyzed in this study are listed in Table 2.

The following background strains were used to construct the strains:

Saccharomyces cerevisiae MaV203 (MAT $\alpha$, leu2-3,112, trp1-901, his3 $\Delta 200$, ade2-101, gal4 $\Delta$, gal80 $\Delta$, SPAL10::URA3, GAL1::lacZ, HIS3UAS GAL1::HIS3@LYS2, can1 ${ }^{R}$, cyh2 ${ }^{R}$ ) (Purchased from Life Technologies).

Saccharomyces cerevisiae PRa18 (MAT $\alpha$, leu2-3,112, trp1-901, his $3 \Delta 200$, ade2-101, gal4 $\Delta$, gal80 $\Delta$, GAL1::lacZ, can1 $^{R}$, cyh2 $\left.{ }^{R}\right)$. Derived from $\mathrm{S}$. cerevisiae MaV203.

Saccharomyces cerevisiae PRa26: MAT $\alpha$, leu2-3,112, trp1-901, his3 $\Delta 200$, ade2-101, gal4 $\Delta$, gal80 $\Delta$, GAL1::lacZ, rad16::KanMX, $\operatorname{can} 1^{R}, \operatorname{cyh} 2^{R}$. Derived from S. cerevisiae PRa18.

Saccharomyces cerevisiae CfB1010 (MATa; ura3-52; his3 11; leu23/112; MAL2-8c; SUC2; are2 $\Delta::$ loxP-KanMX; X-3::tHMG1-P TEF1$\mathrm{P}_{\mathrm{PGK} 1}$-AtATR2). Derived from S. cerevisiae CEN.PK 102-5B. 
Table 1. Plasmids employed in this study, describing whether they lead to chromosomal integration or propagate autonomously in S. cerevisiae.

\begin{tabular}{llll}
\hline Plasmid & \multicolumn{1}{c}{$\begin{array}{c}\text { Expression cassette } \\
\text { (promoter-ORF-terminator) }\end{array}$} & $\begin{array}{l}\text { Maintenance in } \\
\text { S. cerevisiae through }\end{array}$ & Reference \\
\hline pPR4-3vGFP & pSPAL10-3vGFP-tURA3 & CEN/ARS, HIS3 & This study \\
pPR4-sfGFP & pSPAL10-sfGFP-tURA3 & CEN/ARS, HIS3 & This study \\
pCU2-3vGFP & pCUP1dim-3vGFP-tURA3 & CEN/ARS, URA3 & This study \\
pCfB258-CUP1-3vGFP & pCUP1-3vGFP-tCYC1 & Chromosomal integration & This study \\
pCfB258-CUP1-SPO13-3vGFP & pCUP1dim-3vGFP-tCYC1 & Chromosomal integration & This study \\
pDS1U-X2-3vGFP & pTEF1-3vGFP & Chromosomal integration & This study \\
pEXP22 & pADH1-GAL4AD-RalGDS-tADH1 & TRP1 & Life Technologies \\
pEXP32 & pADH1-GAL4DBD-Krev1-tADH1 & LEU2 & Life Technologies \\
pRS413 & - & LEU2 & (Sikorski and Hieter 1989) \\
pRS415 & - & HIS3 & (Sikorski and Hieter 1989) \\
\hline
\end{tabular}

Table 2. Saccharomyces cerevisiae strains analyzed in this study, indicating which plasmids or chromosomal integrations were introduced into the respective parental strains.

\begin{tabular}{llllllll}
\hline Strain name & Promoter & GFP & Plasmid \#1 & Plasmid \#2 & Plasmid \#3 & Integrative plasmid & Parent strain \\
\hline PRa106 & ON & 3vGFP & pPR4-3vGFP & pEXP32 & pEXP22 & - & PRa26 \\
PRa107 & OFF & 3vGFP & pPR4-3vGFP & pRS415 & pEXP22 & - & PRa26 \\
PRa108 & - & - & pRS413 & pRS415 & pEXP22 & - & PRa26 \\
PRa109 & ON & sfGFP & pPR4-sfGFP & pEXP32 & pEXP22 & - & PRa26 \\
PRa110 & OFF & sfGFP & pPR4-sfGFP & pRS415 & pEXP22 & - & PRa26 \\
CK24 & pCUP1 & 3VGFP & - & - & - & pCfB258-CUP1-3vGFP & CfB1010 \\
CK28 & pCUP1dim & 3VGFP & - & - & - & pCfB258-CUP1-SPO13-3vGFP & CfB1010 \\
PRa114 & pTEF1 & 3vGFP & - & - & - & pDS1U-X2-3vGFP & PRa18 \\
\hline
\end{tabular}

\section{Construction of 3vGFP plasmids}

Plasmids were constructed by uracil excision (USER) cloning. The general method for USER cloning was based on agarose gel purification of the PCR products amplified using DNA polymerase $\mathrm{X} 7$ (Nørholm 2010). These were mixed in an equimolar $20 \mu \mathrm{L}$ reaction with $0.5 \mu \mathrm{L}$ USER enzyme (New England Biolabs) and $0.5 \mu \mathrm{L}$ DpnI FastDigest (Thermo Scientific) in FastDigest buffer at $37^{\circ} \mathrm{C}$ for $1-2 \mathrm{~h}$. Following $25 \mathrm{~min}$ at room temperature, $2.5 \mu \mathrm{L}$ reaction was transformed into $E$. coli. Correctly cloned plasmids were identified using restriction analysis and DNA sequencing. The detailed use of oligonucleotides for assembly of all plasmids is described in Supplementary data.

\section{Construction of strains}

Plasmids and DNA for chromosomal targeting was introduced in S. cerevisiae by methods described previously (Gietz and Schiestl 2007). The PRa18 strain was constructed from the MaV203 strain by deletion of SPAL10::URA3 through replacement with a kanMX gene deletion cassette flanked by loxP recombination sites from the pUG6 plasmid as described before (Güldener et al. 1996). DNA flanks to direct homologous recombination of the cassette to the chromosomal locus were generated by PCR on S. cerevisiae MaV203 gDNA spanning a fragment from 5'-CCATTCAACTAACATCACAC to 5'-CCTTCACCATAAATATGCC (upstream flank) and from 5'-CTCACAAATTAGAGCTTC to $5^{\prime}$ CCCATATCCAACTTCCAA (downstream flank). These flanks were cloned to the kanMX gene deletion cassette and transformed into yeast. The kanMX cassette was looped out by heterologous expression of Cre recombinase from the pSH47 plasmid (Güldener et al. 1996). To construct PRa26 subsequently, the chromosomal HIS3 gene within the rad16 locus was deleted using the same kanMX approach. The targeting flanks spanned regions from 5'-AGTTGGTACACCAGTTATACGG to 5'-AAAGCATAGGATACCGAGAAAC (upstream flank) and 5'TGACATCACCCGAAAAGAAGC to 5' - GATTATGGTTACGATGTCGA (downstream flank).

To construct PRa114, the pTEF1-3vGFP construct was chromosomally integrated into the PRa18 strain using divisible selection (Rugbjerg, Myling-Petersen and Sommer 2015). DNA fragments for integration were liberated from the vector $\mathrm{pDS} 1 \mathrm{U}-\mathrm{X} 2$ 3vGFP by digestion with SmiI and transformed into yeast along with empty divisible selection plasmids pDS2 and pDS3 in order to reconstitute the selectable $\mathrm{Ura}^{+}$phenotype.

To construct respectively CK24 and CK28 from the CfB1010 strain, the pCUP1-3vGFP and pCUP1dim-3vGFP were chromosomally integrated by cloning into the EasyClone integrative vectors (Jensen et al. 2014). The DNA fragments for integration were obtained through NotI digestion of the vectors pCfB258-CUP13vGFP and pCfB258-CUP1-SPO13-3vGFP, respectively, followed by agarose gel purification.

\section{Estimation of TEF1-3vGFP fitness cost}

Microtiter cultures of $200 \mu \mathrm{L}$ YPD was inoculated by 100x backdilution of overnight YPD pre-cultures of PRa114 and PRa108, each inoculated from single colonies. The cultures were cultivated in a 96 -well plate at $30^{\circ} \mathrm{C}$ and continuous shaking in an ELx808 plate reader (BioTek), set to measure optical density every $15 \mathrm{~min}$ at $\mathrm{OD}_{630}$. The plate was covered with a BreathSeal 
(Greiner Bio-one) and plastic lid. Growth rates were calculated for all three biological replicates by exponential regression between $\mathrm{OD}_{630}$ and time (hours) during the same $\mathrm{OD}_{630}$ span of exponential growth phase. All $\mathrm{OD}_{630}$ values were initially standardized to the time zero reading to account for differences in seal absorbance.

\section{Cultivations for stability tests}

The PRa114 strain was cultured from a single colony inoculated in $25 \mathrm{~mL}$ YPD medium and cultured at $30^{\circ} \mathrm{C}$ and $250 \mathrm{rpm}$ horizontal shaking in three parallel lineages. By measuring $\mathrm{OD}_{600}$, the number of generations passed was calculated. Approximately $2 \%$ of the culture was passed to fresh medium and grown again until total 25 generations had passed. For comparison between cultured population and reference strain, approximately $25 \mu \mathrm{L}$ of each cell population was inoculated in YPD medium at the same time and cultured at $30^{\circ} \mathrm{C}$ for $16 \mathrm{~h}$ with $250 \mathrm{rpm}$ horizontal shaking.

\section{Fluorescence measurements}

Pre-cultures in selective SC medium were inoculated from single colonies and cultures overnight at $30^{\circ} \mathrm{C}$. From these, $200 \mu \mathrm{L}$ microtiter cultures of selective SC medium were inoculated and cultured at $30^{\circ} \mathrm{C}$ with $300 \mathrm{rpm}$ horizontal shaking in an Innova shaking incubator for $16 \mathrm{~h}$. As cover, the microtiter plates were covered with a BreathSeal (Greiner Bio-one) and a plastic lid.

The cell cultures were diluted approximately 1:100 in FACS flow buffer (BD Biosciences) and analyzed on a LSR Fortessa flow cytometer (BD Biosciences) equipped with a blue laser $(488 \mathrm{~nm})$ and set to measure 10000 cells within a gate defined by forward and side scatter to capture all yeast cells. A FITC filter $(530 / 30 \mathrm{~nm})$ was used to measure GFP fluorescence reporting the area of the measured peaks. The laser voltage was adjusted to optimally utilize the dynamic range of detection. Data were processed and visualized as histograms with FlowJo version 10 (default settings) by overlaying the populations for each particular comparison.

\section{Sequence alignment}

Simple nucleotide and protein sequence alignment was performed using the ClustalO algorithm (Sievers et al. 2011).

\section{RESULTS AND DISCUSSION}

Amplification of fluorescence by tandems of differently encoded GFPs

To amplify the fluorescence signal of a GFP molecule while keeping transcription strength constant, the new 3vGFP protein was

\section{(A)}
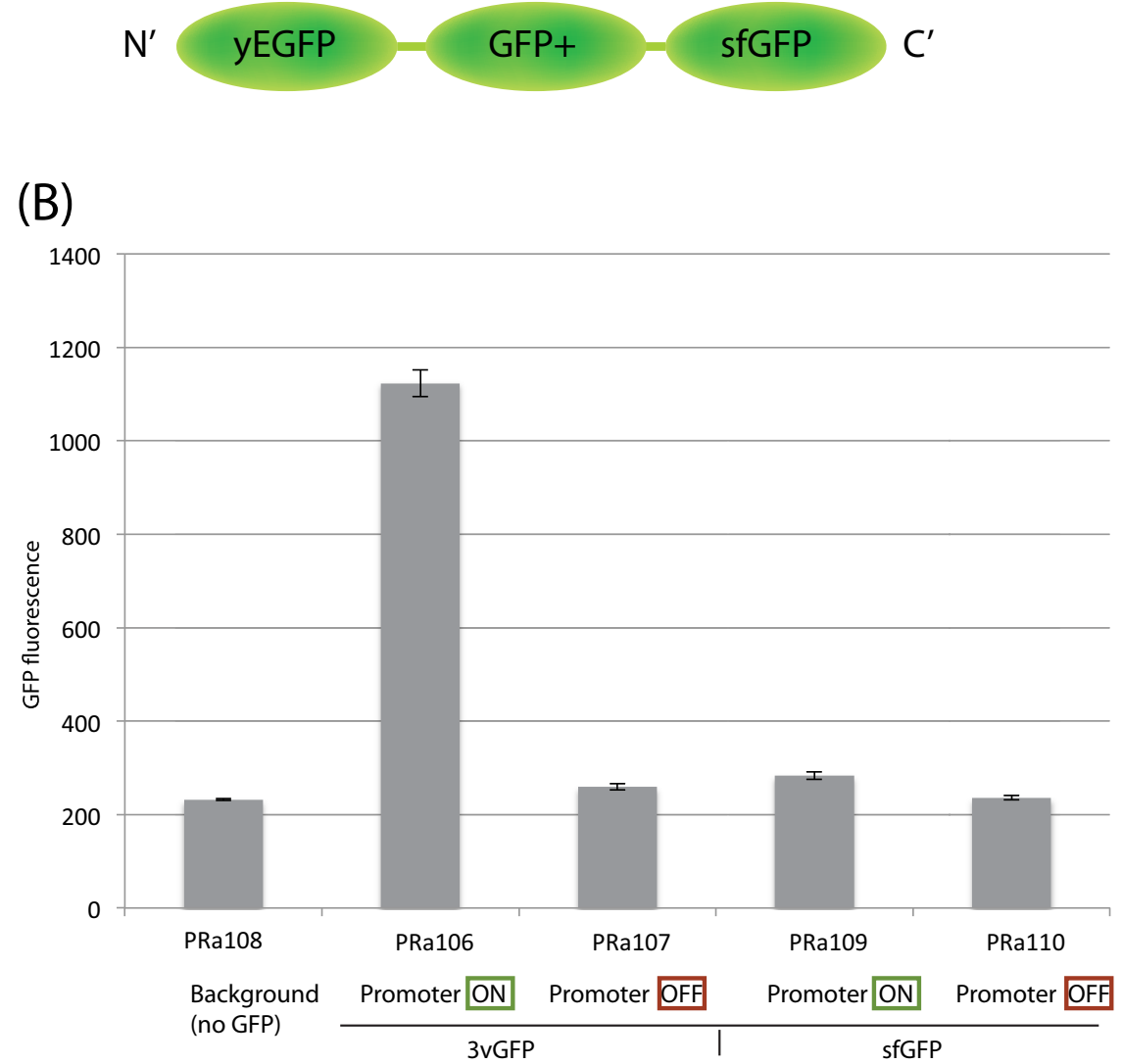

Figure 1. Increased GFP fluorescence signal above autofluorescence level by triple tandem GFP (3vGFP). (A) Internal organization of individual GFP molecules fused as 3vGFP. 3vGFP consists of yeast-enhanced GFP (yEGFP), GFP+ and superfolder GFP. (B) The S. cerevisiae strains carrying 3vGFP allowed the capture of the weak, ON/OFF promoter pSPAL10 unlike strains carrying a single sfGFP. The ON levels with single sfGFP corresponded to the background level of the empty control strain without GFP. The strains are described in detail in Table 2. Error bars depict standard error from biological replicates $(n=3)$. 

5

engineered by fusion of nucleotide sequences encoding yEGFP, GFP+ and sfGFP (Cormack, Bertram and Egerton 1997; Pédelacq et al. 2006) (Fig. 1A). Two glycine residues were introduced as translational linker in each junction. The fluorescence of $3 \mathrm{vGFP}$ was evaluated when expressed from a weak S. cerevisiae hybrid promoter (pSPAL10) (Vidal et al. 1996) based on pSPO13 to mimic low-expression applications (Huang and Schreiber 1997; Harton, Wingler and Cornish 2013). The low-level strength of pSPAL10 is attained by utilizing the UME6 repressor-binding site naturally present within the SPO13 promoter, which allows very low expression levels e.g. useful for control of cell growth. Further, GAL4-binding sites fused $179 \mathrm{bp}$ upstream of start codon provide an upstream activating sequence (UAS), allowing transcription factor-based ON/OFF inputs.

The output fluorescence was first evaluated with single sfGFP (Fig. 1B), which is the individually brightest of the three GFPs tested. However, the fluorescence levels could not be distinguished from the control strain devoid of genes encoding GFP (PRa108). In contrast, the fluorescence of a strain (PRa106) carrying the gene encoding 3vGFP controlled by the same promoter was 3-fold higher than the background level and thus the level of the single sfGFP strain (Fig. 1B).

To test the utility of $3 \mathrm{vGFP}$ as output signal in a synthetic biology setting, we constructed versions of the strain with the pSPAL10 promoter turned OFF. The promoter is activated (ON) when a hybrid GAL4 activation domain binds a cognate hybrid GAL4 DNA-binding domain, which interacts with GAL4binding sites of pSPAL10. The protein-protein interaction domains were based on the known Krev1 and RalGDS interaction domains (Herrmann et al. 1996). However, omitting the DNAbinding domain prevents reconstitution of a functional transactivator (OFF). These ON/OFF effects of present DNA-binding domain remained hidden below the background levels of the sfGFP strains, while observable in strains with 3vGFP as output (Fig. 1B).

\section{Stability towards recombination}

Direct-repeat recombination in mitotic S. cerevisiae is reported to occur at rates between $5.8 \times 10^{-5}$ and $12 \times 10^{-5}$ per cell generation for repeats of several kilo base pair identity (Dornfeld and Livingston 1992). This recombination rate is linearly dependent on identity length at such long segments; however, the rate drops rapidly below the minimal efficient processing segment (MEPS) length at around $250 \mathrm{bp}$ in S. cerevisiae (Jinks-Robertson, Michelitch and Ramcharan 1993). While internal identity of 3vGFP ranges $74-84 \%$ (Fig. 2B), the identical segments are maximally at a 10 -fold shorter length than the MEPS.

To test the recombination stability of $3 \mathrm{vGFP}$, we wanted to measure whether the fluorescence levels originating from $3 \mathrm{vGFP}$ would attenuate following repeated culturing. While the 3vGFP molecule is engineered to limit direct-repeat recombination, long-term cultivation could potentially still lead to this especially if favored by a concurrent fitness advantage. To test stability at high expression level, we therefore also chromosomally integrated 3UGFP under control of the strong promoter from TEF1 i.e. at a level surpassing the intended use of 3vGFP. Expressing 3VGFP from the TEF1 promoter caused a considerate cost in fitness of approximately $15 \%$ in YPD, reducing the growth rate from an average of $0.35-0.30 \mathrm{~h}^{-1}$ compared to the negative control strain PRa108. Following culturing by serial passing (2\%) of liquid cultures for 25 generations of three parallel lineages, single-cell level analysis revealed that the average fluorescence level of the
(A)

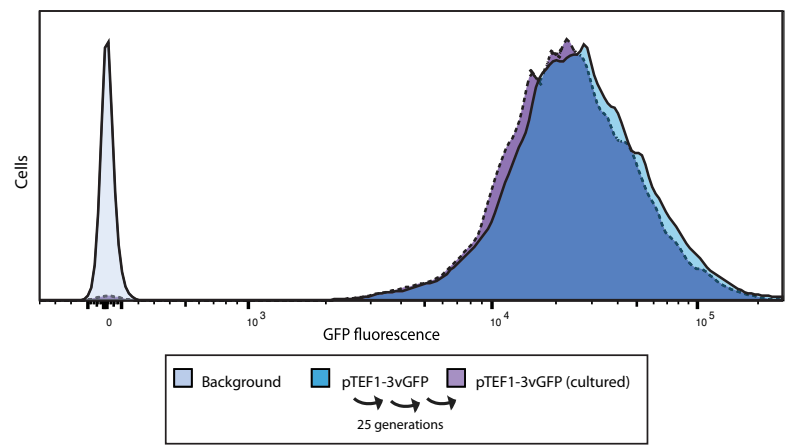

(B)

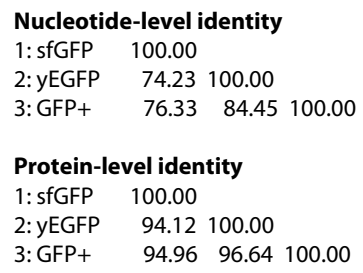

Figure 2. Stability of the triple tandem GFP (3vGFP) towards loop-out recombination. (A) Parallel lineages of a pTEF1-3vGFP S. cerevisiae strain were cultured for 25 generations and re-measured to verify stability towards loop-out recombination, compared to a background strain without GFP. Flow cytometry of representative example shown. Each sample contained 10000 cells. The maxima of the samples are standardized to an equal top point. (B) Sequence identities between the three direct repeats of sequences encoding GFP variants, as calculated by ClustalO.

cell population had diminished by $7 \%$, perhaps due to spontaneous direct-repeat recombination. The single cell-level visualization indicated a slight left shift of the population (Fig. 2A). These results exemplify that direct-repeat recombination can occur within 3vGFP in S. cerevisiae and if selected for, these effects can become significant. However, since 3vGFP is intended for use at levels of low expression, a fitness advantage is not likely to further drive diminished fluorescence at a typical utility of 3vGFP.

\section{Application of 3vGFP to construct an inducible promoter with reduced leakiness}

Inducible promoters are important for development of e.g. synthetic genetic circuits, but the leakiness levels can be problematic in certain uses. To demonstrate the utility of 3vGFP, we therefore wanted to use it as output for genetic reengineering of the popular $\mathrm{Cu}^{2+}$-responsive promoter of S. cerevisiae CUP1. pCUP1 has been employed in many different biotechnological cases (Labbé and Thiele 1999; Scholz et al. 2000; Rugbjerg et al. 2013), but displays considerable baseline activity (leakiness). pCUP1 induction results from elevated $\mathrm{Cu}^{2+}$ concentrations mediated through binding of $\mathrm{Cu}^{2+}$ to the ACE1 transcription factor, which in turn binds to UAS elements of pCUP1 (Huibregtse 1989; Evans, Engelke and Thiele 1990) (elements schematically depicted in Fig. 3A). The leakiness level of pCUP1 measured with 3vGFP corresponded to 2.5-fold the cell autofluorescence (Fig. 3B). Based on the regulatory mechanism of ACE1, we anticipated that trace levels of $\mathrm{Cu}^{2+}$ in the growth medium did not cause this leakiness, but rather assumed this basal transcriptional activity to be ACE1 independent. Accordingly, as strategy we hypothesized that swapping the promoter region downstream of ACE1 UASs for a transcriptionally repressed promoter could 
(A) cup1:

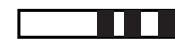

CUP1dim (CUP1-SPO13)

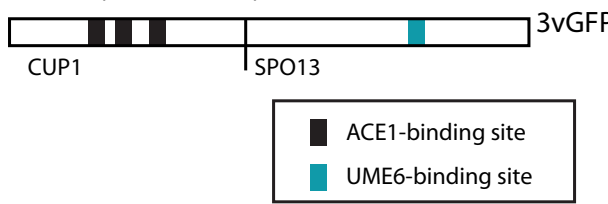

(C)
(B)

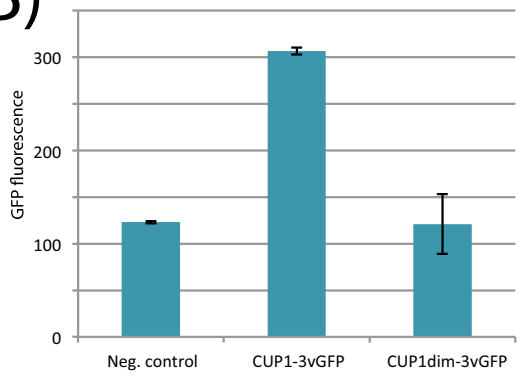

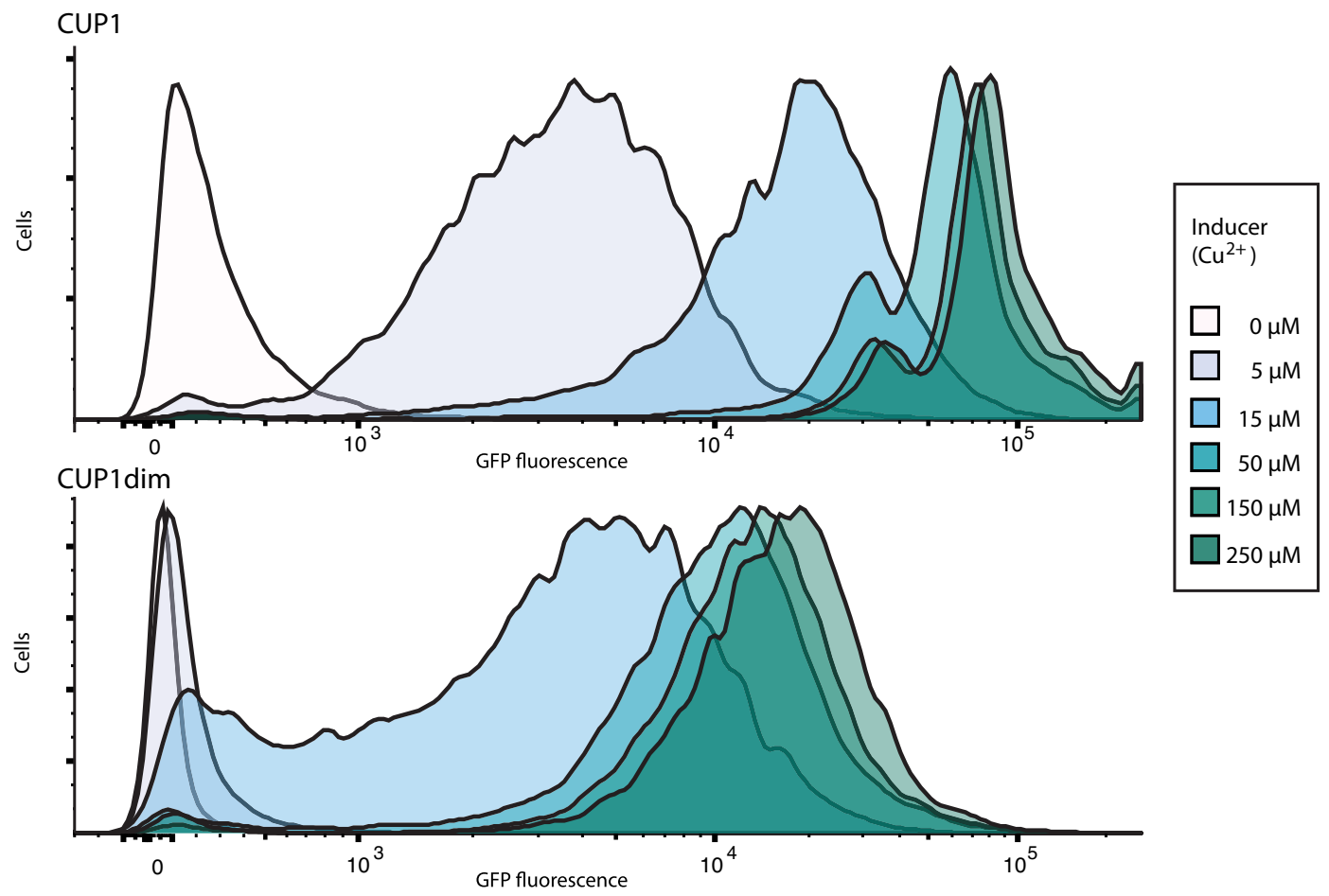

Figure 3. Development of weak $\mathrm{Cu}^{2+}$-responsive promoter through characterization with 3vGFP. (A) Organization of DNA-binding sites for the Cu ${ }^{2+}$-responsive ACE1 activator and UME6 repressor in the wild-type CUP1 promoter and the new dimmed, hybrid promoter pCUP1dim. (B) OFF-level fluorescence measured in absence of $\mathrm{Cu}^{2+}$ demonstrating the lower activity of the new hybrid promoter as captured with 3vGFP. Error bars depict standard error from biological replicates $(n=3)$. (C) Fluorescence of strain populations in response to addition of $\mathrm{Cu}^{2+}$. Flow cytometry of representative example shown. Each sample contained 10000 cells. The maxima of the samples are standardized to an equal top point.

provide attenuation, while maintaining the response to ACE1dependent induction. We therefore combined the upstream region of pCUP1 (-149 to -454$)$ containing three ACE1-binding sites, with part of the S. cerevisiae pSPO13 ( -1 to -157$)$ including its UME6 repressor-binding site (Fig. 3A). This new promoter (pCUP1dim) controlling 3vGFP resulted in fluorescence that was reduced approximately $61 \%$ (before background subtraction) to levels close to the cell autofluorescence (Fig. 3B), while the promoter remained responsive to addition of $\mathrm{Cu}^{2+}$ (Fig. 3C).

The recombination-stabilized tandem GFP described in this study can enable characterization of minimally expressed genes in recombination-efficient organisms such as $\mathrm{S}$. cerevisiae and other yeasts. As shown in this study, 3vGFP allowed characterization of the activation of a weak promoter and accordingly characterization of manipulations taking place at such low expression levels. Further, this particular approach of recombination-stabilizing GFPs with different protein and nu- cleotide sequences can be scaled in number. Recent brighter fluorescent proteins could be applied such as mNeonGreen (Shaner et al. 2013).

In principle, sequence divergence could be generated strictly at nucleotide level through codon optimization of segments encoding the same protein. Codon optimization can however introduce significant effects on the translation efficiencies (Goodman, Church and Kosuri 2013). Another concern may be spurious promoter/RBS activities, which could theoretically cause transcription and translation initiation from locations within the tandem GFP, thus producing truncated tandem proteins. Such situations would complicate the isolation of promoter responses and might require alleviation of the second and third GFP start codon.

An alternative method for assessment of promoter activities could be the use of the fluorescent RNA of the Spinach family, which bypasses the step of translation since the RNA forms 
the fluorescent signal (Paige et al. 2012; Pothoulakis et al. 2014). However, while the technology has potential for synthetic biological use, its general applicability remains to be seen, such as the detection limits for low expression levels. Further relevant, fluorescent in situ hybridization for RNA (RNA FISH) is a technique allowing sensitive detection of transcripts at single-cell level (Zenklusen, Larson and Singer 2008). This alleviates genetic engineering, but entails more sample treatment than for detection of GFP fluorescence.

In this study, a new simple strategy for engineering tandem fluorescent proteins was employed to produce brighter GFP signals with improved stability towards loop-out recombination. GFPs with sequence variation mainly at nucleotide level were translationally linked to form a recombination-stabilized tandem GFP molecule 3vGFP. Such GFPs could be useful for characterizing promoter activities in the range where normal single GFP signals fall below the cell autofluorescence levels. We specifically applied the 3vGFP molecule to characterize the ON/OFF levels of a weak promoter, which was not possible using a single sfGFP, and to develop a new hybrid $\mathrm{Cu}^{2+}$-responsive promoter pCUP1dim with lower leakiness level. The plasmid pCU2-3vGFP encompassing the nucleotide sequence of 3vGFP and pCUP1dim will be deposited at the Addgene repository.

\section{SUPPLEMENTARY DATA}

Supplementary data are available at FEMSYR online.

\section{ACKNOWLEDGEMENTS}

George Church is acknowledged for sfGFP encoded on pJ251GERC (AddGene plasmid 47441).

\section{FUNDING}

This work was supported by the Novo Nordisk Foundation, the European Union Seventh Framework Programme (FP7-KBBE2013-7-single-stage) under grant agreement no. 613745, Promys, and Deutsche Bundesstiftung Umwelt.

Conflict of interest. None declared.

\section{REFERENCES}

Ahn BY, Dornfeld KJ, Fagrelius TJ, et al. Effect of limited homology on gene conversion in a Saccharomyces cerevisiae plasmid recombination system. Mol Cell Biol 1988;8:2442-8.

Ajikumar PK, Xiao W-H, Tyo KEJ, et al. Isoprenoid pathway optimization for Taxol precursor overproduction in Escherichia coli. Science 2010;330:70-4.

Ang J, Harris E, Hussey B. Tuning response curves for synthetic biology. ACS Synth Biol 2013;2:547-67.

Billinton N, Knight AW. Seeing the wood through the trees: a review of techniques for distinguishing green fluorescent protein from endogenous autofluorescence. Anal Biochem 2001;291:175-97.

Blazeck J, Alper HS. Promoter engineering: recent advances in controlling transcription at the most fundamental level. Biotechnol J 2013;8:46-58.

Cormack B, Bertram G, Egerton M. Yeast-enhanced green fluorescent protein (yEGFP): a reporter of gene expression in Candida albicans. Microbiology 1997;143:303-11.

Datta A, Hendrix M, Lipsitch M, et al. Dual roles for DNA sequence identity and the mismatch repair system in the reg- ulation of mitotic crossing-over in yeast. P Natl Acad Sci USA 1997;94:9757-62.

Dornfeld KJ, Livingston DM. Plasmid recombination in a rad52 mutant of Saccharomyces cerevisiae. Genetics 1992;131:261-76.

Evans C, Engelke D, Thiele D. ACE1 transcription factor produced in Escherichia coli binds multiple regions within yeast metallothionein upstream activation site sequences. Mol Cell Biol 1990;10:426-9.

Gahlmann A, Moerner WE. Exploring bacterial cell biology with single-molecule tracking and super-resolution imaging. Nat Reu Microbiol 2014;12:9-22.

Genové G, Glick B, Barth A. Brighter reporter genes from multimerized fluorescent proteins. Biotechniques 2005;39:814-22.

Ghaemmaghami S, Huh W-K, Bower K, et al. Global analysis of protein expression in yeast. Nature 2003;425:737-41.

Gietz RD, Schiestl RH. High-efficiency yeast transformation using the LiAc/SS carrier DNA/PEG method. Nat Protoc 2007;2:31-4.

Goodman D, Church G, Kosuri S. Causes and effects of $\mathrm{N}$-terminal codon bias in bacterial genes. Science (80-) 2013;342:475-9.

Güldener U, Heck S, Fielder T, et al. A new efficient gene disruption cassette for repeated use in budding yeast. Nucleic Acids Res 1996;24:2519-24.

Harton M, Wingler L, Cornish V. Transcriptional regulation improves the throughput of three-hybrid counter selections in Saccharomyces cerevisiae. Biotechnol J 2013;8:1485-91.

Herrmann C, Horn G, Spaargaren M, et al. Differential interaction of the ras family GTP-binding proteins H-Ras, Rap1A, and R-Ras with the putative effector molecules Raf Kinase and Ral-guanine nucleotide exchange factor. $J$ Biol Chem 1996;271:6794-800.

Huang J, Schreiber SL. A yeast genetic system for selecting small molecule inhibitors of protein-protein interactions in nanodroplets. P Natl Acad Sci USA 1997;94:13396-401.

Huh W-K, Falvo JV, Gerke LC, et al. Global analysis of protein localization in budding yeast. Nature 2003;425:686-91.

Huibregtse J. Copper-induced binding of cellular factors to yeast metallothionein upstream activation sequences. P Natl Acad Sci USA 1989;86:65-9.

Jensen NB, Strucko T, Kildegaard KR, et al. EasyClone: method for iterative chromosomal integration of multiple genes in Saccharomyces cerevisiae. FEMS Yeast Res 2014;14:238-48.

Jinks-Robertson S, Michelitch M, Ramcharan S. Substrate length requirements for efficient mitotic recombination in Saccharomyces cerevisiae. Mol Cell Biol 1993;13:3937-50.

Labbé S, Thiele D. Copper ion inducible and repressible promoter systems in yeast. Methods Enzymol 1999;306:145-53.

Li G-W, Xie XS. Central dogma at the single-molecule level in living cells. Nature 2011;475:308-15.

Lichten CA, White R, Clark IBN, et al. Unmixing of fluorescence spectra to resolve quantitative time-series measurements of gene expression in plate readers. BMC Biotechnol 2014;14:11.

Miyawaki A. Proteins on the move: insights gained from fluorescent protein technologies. Nat Rev Mol Cell Bio 2011;12:656-68.

Nevoigt E, Kohnke J, Fischer CR, et al. Engineering of promoter replacement cassettes for fine-tuning of gene expression in Saccharomyces cerevisiae. Appl Environ Microb 2006;72:5266-73.

Newman JRS, Ghaemmaghami S, Ihmels J, et al. Single-cell proteomic analysis of $\mathrm{S}$. cerevisiae reveals the architecture of biological noise. Nature 2006;441:840-6.

Nørholm MHH. A mutant Pfu DNA polymerase designed for advanced uracil-excision DNA engineering. BMC Biotechnol 2010;10:21. 
Paige JS, Nguyen-Duc T, Song W, et al. Fluorescence imaging of cellular metabolites with RNA. Science 2012;335:1194.

Pédelacq J-D, Cabantous S, Tran T, et al. Engineering and characterization of a superfolder green fluorescent protein. Nat Biotechnol 2006;24:79-88.

Pothoulakis G, Ceroni F, Reeve B, et al. The spinach RNA aptamer as a characterization tool for synthetic biology. ACS Synth Biol 2014;3:182-7.

Raj A, van Oudenaarden A. Single-molecule approaches to stochastic gene expression. Annu Rev Biophys 2009;38:255-70.

Rayssiguier C, Thaler DS, Radman M. The barrier to recombination between Escherichia coli and Salmonella typhimurium is disrupted in mismatch-repair mutants. Nature 1989;342: 396-401.

Rugbjerg P, Myling-Petersen N, Sommer M. Flexible metabolic pathway construction using modular and divisible selection gene regulators. Metab Eng 2015;31:189-97.

Rugbjerg P, Naesby M, Mortensen UH, et al. Reconstruction of the biosynthetic pathway for the core fungal polyketide scaffold rubrofusarin in Saccharomyces cerevisiae. Microb Cell Fact 2013;12:31.

Scholz O, Thiel A, Hillen W, et al. Quantitative analysis of gene expression with an improved green fluorescent protein. Eur J Biochem 2000;267:1565-70.
Shaner NC, Lambert GG, Chammas A, et al. A bright monomeric green fluorescent protein derived from Branchiostoma lanceolatum. Nat Methods 2013;10:407-9.

Sievers F, Wilm A, Dineen D, et al. Fast, scalable generation of high-quality protein multiple sequence alignments using Clustal Omega. Mol Syst Biol 2011;7:539.

Sikorski RS, Hieter P. A system of shuttle vectors and yeast host strains designed for efficient manipulation of DNA in Saccharomyces cerevisiae. Genetics 1989;122:19-27.

Snapp EL. Fluorescent proteins: a cell biologist's user guide. Trends Cell Biol 2009;19:649-55.

Vidal M, Brachmann RK, Fattaey A, et al. Reverse two-hybrid and one-hybrid systems to detect dissociation of proteinprotein and DNA-protein interactions. P Natl Acad Sci USA 1996;93:10315-20.

Xie XS, Choi PJ, Li G-W, et al. Single-molecule approach to molecular biology in living bacterial cells. Annu Rev Biophys 2008;37:417-44.

Yu J, Xiao J, Ren X, et al. Probing gene expression in live cells, one protein molecule at a time. Science (80-) 2006;311: 1600-3.

Zenklusen D, Larson DR, Singer RH. Single-RNA counting reveals alternative modes of gene expression in yeast. Nat Struct Mol Biol 2008;15:1263-71. 